\title{
Brain stem auditory evoked responses in chronic alcoholics
}

\author{
Y-W CHAN, JG MCLEOD, RR TUCK, PA FEARY \\ From the Department of Medicine, University of Sydney, and Department of Neurology, Royal Prince Alfred \\ Hospital, Sydney, Australia
}

SUMMARY Brain stem auditory evoked responses (BAERs) were performed on 25 alcoholic patients with Wernicke-Korsakoff syndrome, 56 alcoholic patients without Wernicke-Korsakoff syndrome, 24 of whom had cerebellar ataxia, and 37 control subjects. Abnormal BAERs were found in $48 \%$ of patients with Wernicke-Korsakoff syndrome, in $25 \%$ of alcoholic patients without Wernicke-Korsakoff syndrome but with cerebellar ataxia, and in $13 \%$ of alcoholic patients without Wernicke-Korsakoff syndrome or ataxia. The mean value of the I-V interval was prolonged in all patient groups. There were more patients with prolonged I-V and I-III intervals in the Wernicke-Korsakoff syndrome group than in the group without the syndrome. The I-III interval was prolonged in $32 \%$ of those with Wernicke-Korsakoff syndrome but in only $6 \%$ of alcoholics without the syndrome. These abnormalities improved following thiamine treatment and abstinence from alcohol. The presence of prolonged I-III interval in an alcoholic should raise the possibility of Wernicke's encephalopathy.

The Wernicke-Korsakoff syndrome is a neurological disorder usually encountered in alcoholics and caused by thiamine deficiency. ${ }^{1}$ In the acute phase (Wernicke's encephalopathy), the signs are nystagmus, ophthalmoplegia, confusion and ataxia, all of which improve with administration of thiamine. ${ }^{1}$ Many affected patients are left with a severe deficit in short term memory (Korsakoff psychosis). The large discrepancy between the number of cases diagnosed clinically and pathologically ${ }^{2-4}$ suggests that the diagnosis of Wernicke's encephalopathy is frequently overlooked during life.

The lesions in Wernicke-Korsakoff syndrome are primarily in the grey matter of the brain stem and diencephalon, ${ }^{4-6}$ and also brain stem abnormalities have been demonstrated pathologically in alcoholic cerebellar degeneration. ${ }^{7}$ Lesions in these regions of the brain are difficult to localise neuroradiologically but brain stem abnormalities may sometimes be detected by brain stem auditory evoked responses (BAERs). ${ }^{89}$ Abnormal BAERs have been reported in chronic alcoholics including those with Wernicke-Korsakoff syndrome ${ }^{10}$ but the number of

Address for reprint requests: Dr JG McLeod, Department of Medicine, University of Sydney, NSW 2006, Australia.

Received 28 December 1984 and in revised form 27 March 1985. Accepted 5 April 1985 cases studied has been small. Serial studies correlating BAER with clinical recovery have not been described in Wernicke-Korsakoff syndrome.

We report a study of the BAERs in 25 alcoholic patients with Wernicke-Korsakoff syndrome, 16 of whom were followed up for 6 months, and in 56 alcoholic patients who had no history of Wernicke-Korsakoff syndrome. The aim of our study was to examine the incidence and nature of BAER abnormalities in alcoholic patients with and without Wernicke-Korsakoff syndrome and to evaluate the effect of therapeutic measures and complete abstinence from alcohol on the BAER.

\section{Methods}

\section{SUBJECTS}

Controls

The control subjects were 37 male and 40 female volunteers whose ages ranged from 18 to 69 years (mean 43.5 years (males); 46.5 years (females)). Informed consent, and drinking and medical histories were obtained. Only healthy subjects who abstained or drank less than $20 \mathrm{~g} /$ day and were not on drug treatment were accepted for the study.

Chronic alcoholics without Wernicke-Korsakoff syndrome The group consisted of 56 males who had a history of an alcohol consumption of 50 to $200 \mathrm{~g} /$ day for 10 to 30 years 
but who had no history or clinical features of WernickeKorsakoff, syndrome. Ages ranged from 25 to 63 years (mean 47.5 years). They were further divided into two groups; group I ( 32 patients) had no evidence of cerebellar degeneration; group II ( 24 patients) had truncal and gait ataxia compatible with alcoholic cerebellar degeneration. ${ }^{17}$ A minimum abstinent period of 2 weeks was required before BAERs were recorded.

\section{Chronic alcoholics with Wernicke-Korsakoff syndrome}

The group consisted of 25 alcoholic patients admitted to hospital in whom the clinical diagnosis of WernickeKorsakoff syndrome was confirmed by neurological assessment. Their ages ranged from 42 to 70 years (mean 53.5 years); 21 were males and four were females. They had a drinking history ranging from 10 to 35 years with a daily intake of 50 to $250 \mathrm{~g}$. The clinical diagncsis of Wernicke's encephalopathy was made in 17 patients by the presence of mental confusion, ophthalmoplegia, nystagmus and ataxia. In the other eight patients there was no obvious mental confusion reported on routine clinical examination but ophthalmoplegia and ataxia were present and responded promptly to thiamine. On subsequent testing all patients had some degree of short term memory loss; seven were unable to recall any of three unrelated words after 3 minutes and were diagnosed as having Korsokoffs psychosis.

The initial BAER was performed within 14 to 30 days (mean 25 days) of admission to hospital during which time they were completely abstinent of alcohol. Those who had concurrent illnesses that might affect brain stem function (for example severe head injury, cerebrovascular diseases and hypothermia) were excluded from the study.

\section{AUDIOLOGICAL EXAMINATION}

The hearing of the control and patient groups was assessed audiologically. Examination with an auroscope after removal of ear wax was performed on each subject followed by a pure tone audiogram. Those who had severe peripheral hearing loss were not accepted for BAER studies. The click threshold of eaeh ear was checked before the BAER. No BAER was performed if the threshold exceeded $20 \mathrm{~dB}$ HL.

\section{BRAIN STEM AUDITORY EVOKED RESPONSES (BAERS)}

BAER recordings were performed in an isolated room using a Medelec modified averaging system. The stimulus was a wide band $100 \mu$ s click, generated from an Amplaid FRA research audiometer which was controlled by a Medelec AS6 trigger unit. Clicks were presented monaurally through earphones at a repetition rate of $12 \mathrm{~Hz}$, and at an intensity of 65 to $75 \mathrm{~dB}$ above the subjective click threshold of the ear. The rarefaction click polarity was employed in this study.

Percutaneous needle electrodes were placed at the vertex and both ear lobes, the ground electrode being placed under the chin. Ipsilateral and contralateral recordings with reference to the vertex were taken simultaneously. The contralateral ear received a masking white noise whilst clicks were presented to the ipsilateral ear. The electrodes were connected to Medelec AA6 MK3 amplifier which had a gain of 100000 and a bandpass of $100 \mathrm{~Hz}$ and $3 \cdot 2 \mathrm{~Hz}$.
The output of the amplifier was led to a 1024 point analog averager (Medelec DAV6) which was gated on during the first $10 \mathrm{~ms}$ following each click stimulus. A total of 1000 responses were averaged for each record. At least two trials were conducted for each run in order to ensure the reproducibility of the results. The responses were recorded on an $\mathrm{X}-\mathrm{Y}$ plotter, in which vertex positivity or ear lobe negativity was indicated by an upward deflection of the tracing."

Waves I to $\mathrm{V}$ were identified according to the conventional methods. Their respective latencies were measured from the stimulus artefact to the peaks, to the nearest $0.1 \mathrm{~ms}$. The interpeak latencies I-III, III-V and I-V were then calculated from the absolute latencies. If there was difficulty in identifying wave $I$, an electrode inserted into the anterior wall of the external auditory canal usually enabled it to be recognised.

Our criteria for defining an abnormal BAER are: (a) Prolongation of one or more of the interpeak latencies I-III, III-V and I-V beyond the mean +3 SD values of the normal subjects, (b) Absence of wave III or wave $V$ in the presence of a clearly defined wave I. If wave I could not be identified, the result was not included for analysis. Prolongation of absolute latency was not used as a criterion for abnormality because absolute latencies are more affected by peripheral hearing losses than the interpeak latencies. ${ }^{12}$

FOLLOW UP STUDIES

The Wernicke-Korsakoff syndrome-alcoholic patients were discharged to a convalescent hospital after the acute phase. Vitamin therapy, adequate nutrition and complete abstinence from alcohol were ensured while staying in hospital. Any patients who were discharged before 6 months and who resumed alcohol-drinking were not tested further. Patients exhaled into an alcohol meter before the recording to ensure that no tests were done under the influence of recent alcohol intake. ${ }^{13}$ We were able to follow up 16 patients with a second BAER at 6 months.

\section{STATISTICS}

The data were stored in a disc using a VAX 780 minicomputer and were analysed by means of the Scientific Information Retrieval and Statistical Packages of Social Science (SPSS) software packages. The non-paired $t$ test was used to compare the means between groups and the paired $t$ test to compare the initial and follow-up results in the Wernicke-Korsakoff syndrome-alcoholic group. The differences between means was considered significant when $\mathrm{p}<0.05$. The chi-square test was used to compare the percentages of patients with abnormal BAERs between the Wernicke-Korsakoff syndrome and nonWernicke-Korsakoff syndrome groups. In text, tables and figures, mean values are expressed with one standard deviation.

\section{Results}

\section{Controls}

Waves I, III and V were present in the BAERs of all the controls (fig 1). Since there were no significant differences in latencies between ears, the data for 


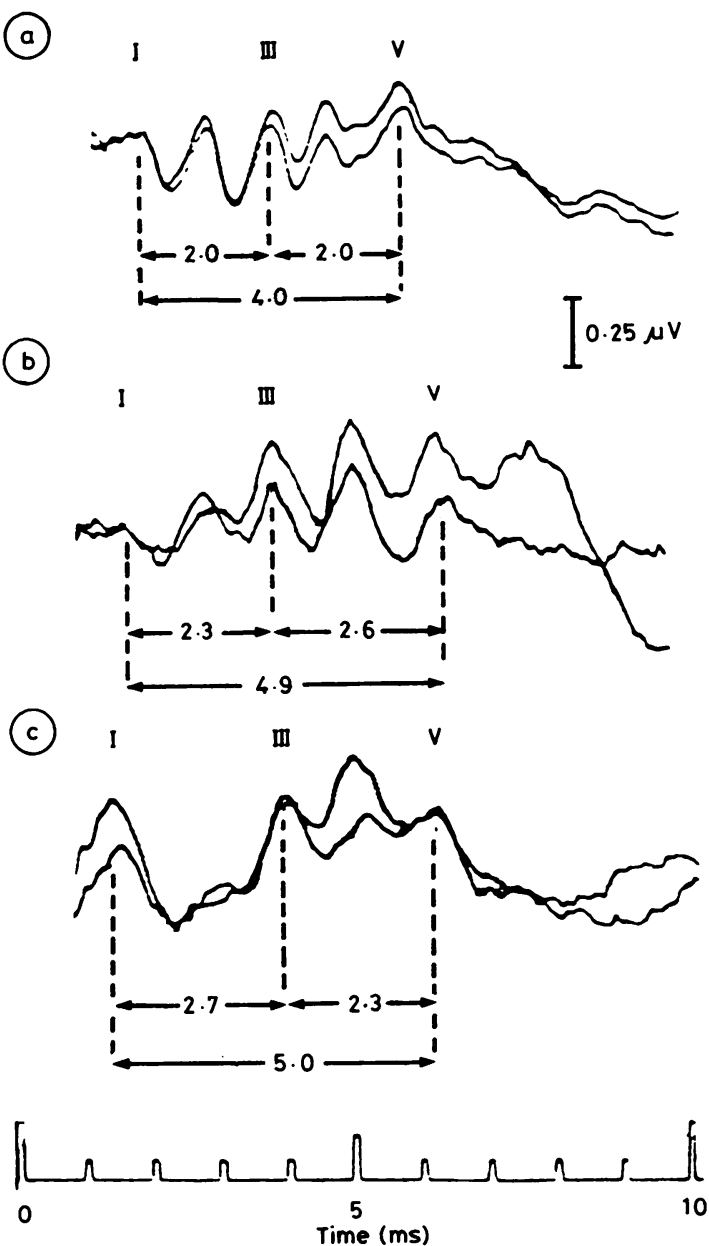

Fig 1 BAERs in a control subject (a), a non-Wernicke-Korsakoff syndrome alcoholic patient with cerebellar ataxia (b) and an alcoholic patient with Wernicke-Korsakoff syndrome (c). Note the prolongation of the $I-V$ and $I I I-V$ interpeak latencies in $B$ and the prolongation of the I-V and I-III interpeak latencies in C Stimulus to right ear with rarefaction clicks at a repetitive rate of 12 per second, intensity $70 \mathrm{~dB} S L$. both ears were pooled. The mean $+3 \mathrm{SD}$ in males and females respectively for the $\mathrm{I}-\mathrm{V}$ interval was $4.6 \mathrm{~ms}$ and $4.6 \mathrm{~ms}$; for the I-III interval was $2.6 \mathrm{~ms}$ and $2.5 \mathrm{~ms}$; and for the III-V interval was $2.4 \mathrm{~ms}$ and $2.3 \mathrm{~ms}$. There was no significant effect of age on the interpeak latencies.

\section{Chronic alcoholics without Wernicke-Korsakoff syndrome}

Group I Of the 32 patients, only four (13\%) patients had abnormal BAERs. There were two patients with prolonged I-III intervals, one patient with prolonged III-V interval, and one with absent wave III and V (table 1). There was significant prolongation of the I-V interval for the whole group ( $p$ $<0.05)$. The I-III and III-V intervals were also prolonged but the increase was not significant (table 2, fig 2).

Group II Of the 24 patients, six (25\%) had abnormal BAERs. There were three patients with prolonged III-V and I-V intervals, one with a prolonged I-III interval, one with a prolonged III-V interval and one with an absent wave $\mathrm{V}$ (table 1 , fig 1). The I-V and III-V intervals were significantly prolonged ( $p<0.005$ and $p<0.02$ respectively). The increase in I-III interval was not significant (table 2, fig 2).

Chronic alcoholics with Wernicke-Korsakoff syndrome Of the 25 patients $(21$ males and four females), 12 (48\%) had abnormal BAERs. Abnormalities were found in all the interpeak latencies, especially the I-V and I-III intervals. There were six patients with a prolonged $\mathrm{I}-\mathrm{V}$ interval, eight patients with a prolonged I-III interval, two with a prolonged III-V interval and one with absent waves III and V (table 1, fig 1). In the 21 males the mean latencies of both the I-V and I-III intervals were significantly prolonged $(\mathrm{p}<0.01$ and $\mathrm{p}<0.002$ respectively). The increase in the III-V interval was not significant (table 2, fig 2).

The I-III interval was prolonged in $32 \%$ of

Table 1 BAER abnormalities in Wernicke-Korsakoff syndrome and non-Wernicke-Korsakoff syndrome subjects

\begin{tabular}{|c|c|c|c|}
\hline \multirow{2}{*}{$\begin{array}{l}\text { Abnormalities of interpeak } \\
\text { latencies }\end{array}$} & \multicolumn{2}{|c|}{ Non-Wernicke-Korsakoff syndrome alcoholic } & \multirow{2}{*}{$\begin{array}{l}\text { Wernicke-Korsakoff } \\
\text { syndrome-alcoholics } \\
n=25\end{array}$} \\
\hline & Group I $n=32$ & Group II $n=24$ & \\
\hline $\begin{array}{l}\text { Prolonged I-V only } \\
\text { Prolonged I-III and I-V } \\
\text { Prolonged III-V and I-V } \\
\text { Prolonged I-III only } \\
\text { Prolonged III-V only } \\
\text { Absent IIIV } \\
\text { Total no (\%) }\end{array}$ & $\begin{array}{l}0 \\
0 \\
0 \\
2 \\
1 \\
1 \\
4(13 \%)\end{array}$ & $\begin{array}{l}0 \\
0 \\
3 \\
1 \\
1 \\
1 \\
6(25 \%)\end{array}$ & $\begin{array}{l}1 \\
4 \\
1 \\
4 \\
1 \\
1 \\
12(48 \%)\end{array}$ \\
\hline
\end{tabular}


Table 2 BAER in control subjects and chronic alcoholics

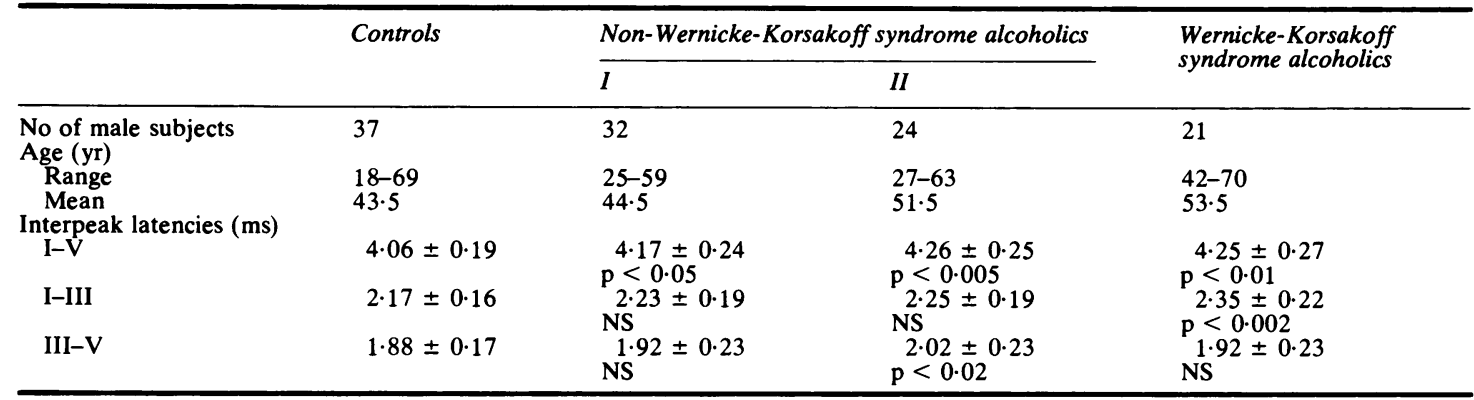

patients with Wernicke-Korsakoff syndrome but in only $6 \%$ of those without Wernicke-Korsakoff syndrome. The I-V interval was prolonged in $24 \%$ of Wernicke-Korsakoff syndrome and $12 \%$ of nonWernicke-Korsakoff syndrome group II and none of non-Wernicke-Korsakoff syndrome group I. The percentages of patients with prolonged I-III and I-V intervals were significantly higher in Wernicke-Korsakoff syndrome group than in non-Wernicke-Korsakoff syndrome groups $\left(\chi^{2}=\right.$ 8.3 and $4.34, p<0.01$ and $p<0.05$ respectively).

\section{Follow up studies}

Mental confusion, ophthalmoplegia and nystagmus were no longer present at follow up. All were able to

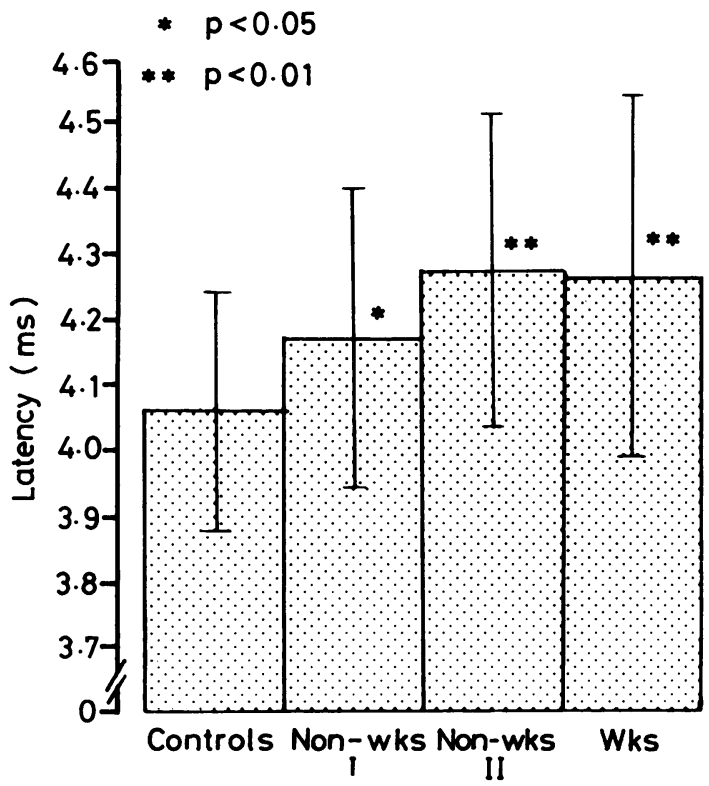

Fig 2 Interpeak latency $I-V$ in controls and chronic alcoholics. Bars represent mean latency, with one standard deviation.
Table 3 Follow-up studies of BAERs in Wernicke-Korsakoff syndrome

\begin{tabular}{llll}
\hline & 1st test & 2nd test & \\
\hline No of subjects & 16 & 16 & \\
Sex (M:F) & $15: 1$ & $15: 1$ & \\
Abnormal BAER & $8(50 \%)$ & $2(13 \%)$ & \\
Interpeak latencies & & & \\
I-V & $4 \cdot 29 \pm 0.27$ & $4.18 \pm 0.27$ & $\mathrm{p}<0.05$ \\
I-III & $2 \cdot 38 \pm 0.20$ & $2.26 \pm 0.22$ & $\mathrm{p}<0.01$ \\
III-V & $1.94 \pm 0.24$ & $1.90 \pm 0.18$ & NS \\
\hline
\end{tabular}

walk without assistance although some degree of 0 gait ataxia was present in the majority including the two whose BAERs remained abnormal.

Of the 16 patients with Wernicke-Korsakoff syn-o drome followed up for 6 months, eight $(50 \%)$ had abnormal BAERs at the initial study, but only two $(13 \%)$ had abnormal BAERs at the second exami-o nation. All the mean interpeak latencies were shorter at the second examination. These were significant in the cases of the I-III and I-V intervals $(\mathrm{p}<0.01, \mathrm{p}<0.05$ respectively) (table 3$)$.

\section{Discussion}

The diagnosis of Wernicke-Korsakoff syndrome was made in 17 patients by the presence of confusion, ophthalmoplegia, nystagmus and ataxia. In eight patients there was no confusion but ophthalmoplegia and ataxia were present and responded promptly to thiamine. According to Victor et al, ${ }^{1}$ confusion is absent in $10 \%$ of patients with pathologically proven Wernicke-Korsakoff syndrome. The chronic alcoholic group consisted of alcoholic patients who had no history of the syndrome. Group I patients had no gait disturbance while group II had evidence of cerebellar involvement.

The study showed that among the three alcoholic groups, the Wernicke-Korsakoff syndrome group had the highest incidence of abnormal BAERs (48\%). The non-Wernicke-Korsakoff syndrome 
group II patients had an incidence of $25 \%$ abnormalities and the non-Wernicke-Korsakoff syndrome group I patients had $13 \%$ abnormalities. In a similar study by Chu et al ${ }^{10}$ of chronic alcoholic patients, those with cerebellar degeneration had the highest incidence of abnormalities (83\%) while in patients with either Wernicke-Korsakoff syndrome or alcoholic dementia, the incidence was $50 \%$. However, in their report the number of patients studied in each of the subgroups was relatively small (less than six patients). In another study by Rosenhamer and Silfverskiold ${ }^{14} 10$ of $13(77 \%)$ alcoholic patients who had slow tremor and cerebellar ataxia had abnormal BAERs. However, their criterion for abnormality was mean $+2 \mathrm{SD}$. If the mean $+3 \mathrm{SD}$ value is taken as the upper limit of normal, the incidence of abnormalities in their study is $23 \%$ (three out of 13), a figure which is similar to that of our non-Wernicke-Korsakoff syndrome group II patients.

The BAER reflects the functional integrity of the brain stem auditory pathways. Although some uncertainty still exists about the origin of the waves that constitute the BAER the most likely generators are: wave I, acoustic nerves; wave II, cochlear nucleus; wave III, superior olivary complex; wave IV, ventral nucleus of lateral lemniscus and wave $V$, inferior colliculus. ${ }^{15}$ Analysis of the type of BAER abnormalities detected in chronic alcoholics may provide evidence about the sites of the brain stem lesions. In Wernicke-Korsakoff syndrome the acute lesions are characterised by proliferative changes in the blood vessels, perivascular haemorrhages and loss of myelin and axons. These lesions occur in the brain stem auditory pathways and in the adjacent structures. The fact that the abnormal signs and the abnormalities in the BAERs in Wernicke-Korsakoff syndrome may be reversed by thiamine suggests that the initial disturbances in neurological function may be biochemical. Begleiter et al ${ }^{16}$ studied 17 chronic alcoholic patients and found a delay in all the mean interpeak latencies and in the absolute latencies of waves II, III, IV and V. These findings indicate widespread dysfunction from the cochlear nucleus to the inferior colliculus. Our study has also shown that the mean I-V interval was prolonged in all groups of chronic alcoholic patients. However prolongation of the I-V interval was approximately twice as frequent in the Wernicke-Korsakoff syndrome group as in the non-Wernicke-Korsakoff syndrome group. Prolongation of the I-III intervals was more than five times more frequent in patients with Wernicke-Korsakoff syndrome than in alcoholics without Wernicke-Korsakoff syndrome, a finding which raises the possiblity of damage in the peripheral pathway including the acoustic nerve in
Wernicke-Korsakoff syndrome. Chu et al ${ }^{10}$ found that the III-V interval was prolonged in patients with alcoholic cerebellar degeneration and their finding is supported by the present study in which the III-V interval was increased but there was no significant prolongation of the I-III interval. Rosenhamer et al,${ }^{14}$ however, found prolongation of both the I-III and III-V intervals in 13 patients with cerebellar ataxia. Pathological changes in the brain stem were reported in alcoholic cerebellar degeneration by Victor $e t a^{7}$ and included loss of cells of the inferior olives, vestibular nuclei and nucleus pontis.

Our study has demonstrated potentially reversible abnormalities of BAERs in chronic alcoholic patients, particularly those with WernickeKorsakoff syndrome in whom the changes are most evident as an increase in the I-III interpeak latency.

The work was supported by grants from the Australian Associated Brewers and the Bushell Trust. Dr Chan was in receipt of a Sir Robert Black Trust Scholarship from Hong Kong. The authors thank Dr JC Walsh, Dr C Yiannikas and Dr GM Halmagyi for invaluable advice.

\section{References}

' Victor MA, Adams RD, Collins GH. The WernickeKorsakoff Syndrome. Philadelphia: FA Davies, 1971.

${ }^{2}$ Victor M, Laureno R. Neurologic complications of alcohol abuse: epidemiological aspects. In: Schoenberg BS, ed. Advances in Neurology, Vol 19. Neurological Epidemiology: Principles and Clinical Application. New York: Raven Press, 1978:603-18.

${ }^{3}$ Torvik A, Lindboe C, Rodge S. Brain lesions in alcoholics. A neuropathological study with clinical correlations. J Neurol Sci 1982;56:233-48.

${ }^{4}$ Harper C. The incidence of Wernicke's encephalopathy in Australia - a neuropathological study of 131 cases. J Neurol Neurosurg Psychiatry 1982;46:593-8.

${ }^{5}$ Lynch M. Brain lesions in chronic alcoholics. Arch Pathol 1961;69:342-53.

' Harper C. Wernicke's encephalopathy: a more common disease than realised. J Neurol Neurosurg Psychiatry 1979;42: 226-31.

7 Victor M, Adams RD, Mancall EL. A restricted form of cerebellar cortical degeneration occurring in alcoholic patients. Arch Neurol 1959;1:577-688.

${ }^{8}$ Starr A Achor J. Auditory brain stem responses in neurological diseases. Arch Neurol 1975;32:761-8.

9 Stockard J, Rossiter V. Clinical and pathologic correlates of brain stem auditory response abnormalities. Neurology (Minneap) 1977;27:316-25.

${ }^{10} \mathrm{Chu} \mathrm{N}$, Squires K, Starr A. Auditory brain stem responses in chronic alcoholic patients. Electroencephalogr Clin Neurophysiol 1982;54:418-25.

" Jewett D, Romano M, Williston J. Human auditory evoked potentials: possible brain stem components detected on the scalp. Science 1970;167:1517-8. 
${ }^{12}$ Rosenhamer H, Lindstrom B, Lundborg T. On the use of click-evoked electric brain stem responses in audiological diagnosis. III. Latencies in cochlear hearing loss. Scand Audiol 1981;10:3-11.

${ }^{13}$ Church M, William H. Dose and time dependent effects of ethanol on brain stem auditory evoked responses in young adult males. Electroencephalogr Clin Neurophysiol 1982;54:161-74.
${ }^{14}$ Rosenhamer H, Silfverskiold B. Slow tremor and delayed brain stem auditory evoked responses in alcoholics. Arch Neurol 1980;37:293-6.

${ }^{15}$ Chiappa KH, Ropper AH. Evoked potentials in clinical medicine. N Engl J Med 1982;306:1140-50.

${ }^{16}$ Begleiter H, Porjesz B, Chou CL. Auditory brainstem potentials in chronic alcoholics. Science 1981; 211:1064-6. 